\title{
Monte Carlo simulations of electron photoemission from plasmon-enhanced bialkali photocathode
}

\author{
Zenggong Jiang $\odot$, Qiang Gu๑," and Xudong Li \\ Shanghai Advanced Research Institute, Chinese Academy of Sciences, \\ Shanghai 201210, People's Republic of China \\ Erdong Wang, Mengjia Gaowei, and Wei Liu® \\ Brookhaven National Laboratory, Upton, New York 11794, USA
}

(Received 11 March 2020; accepted 12 February 2021; published 25 March 2021)

\begin{abstract}
Photocathodes based on bialkali antimonides have great potential in the field of high-brightness electron sources. In this paper, the influence of incident light absorption on the performance of $\mathrm{K}_{2} \mathrm{CsSb}$ photocathodes was studied using Monte Carlo simulations. The surface plasmon polaritons (SPPs) were first introduced into $\mathrm{K}_{2} \mathrm{CsSb}$ photocathodes employing a nanopattern structure to improve the effective absorption of incident light and adjust the initial distribution of the photoelectrons. To evaluate the effect of SPPs on the photocathode performance, photoemission from such photocathodes was modeled using Monte Carlo simulations. Simulations show the quantum efficiency of the $\mathrm{K}_{2} \mathrm{CsSb}$ photocathode increases by 2-3 times that of the photocathode without SPPs. Moreover, the thermal emittance of the photocathode remains unchanged. These results are crucial to the future development of high-brightness electron beams.
\end{abstract}

DOI: 10.1103/PhysRevAccelBeams.24.033402

\section{INTRODUCTION}

High-performance photocathodes have become the enabling technology for advanced light sources and accelerators, such as x-ray free electron laser, energy recovery linac, and ultrafast electron diffraction [1-3]. Quantum efficiency $(\mathrm{QE})$, which characterizes the photoelectron emission capability, is an important parameter for evaluating the performance of photocathodes. Photocathodes with high QEs can effectively reduce the power requirement of the photocathode laser system, thereby reducing the cost of the photocathode laser system [4]. They can also reduce the operational risk of the high-power photocathode laser damaging the optical elements of the laser system. Moreover, they effectively increase their operational lifetime and reduce interruptions in the continuous operation of a facility [5]. A higher QE also allows the use of photocathode lasers with lower photon energies, reducing the thermal emittance of the photocathode [6,7].

The $\mathrm{K}_{2} \mathrm{CsSb}$ photocathode with high $\mathrm{QE}$ and low thermal emittance driven by visible light plays an important role in the field of the high-repetition-rate, high-brightness

\footnotetext{
*Corresponding author. guqiang@sinap.ac.cn

Published by the American Physical Society under the terms of the Creative Commons Attribution 4.0 International license. Further distribution of this work must maintain attribution to the author(s) and the published article's title, journal citation, and DOI.
}

electron sources. At present, a photocathode is usually prepared by depositing a thin film of $\mathrm{K}_{2} \mathrm{CsSb}$ on a substrate through thermal deposition [8], molecular beam epitaxy [9], and sputter deposition [10]. With optimized preparation processes, the typical QE of multialkali photocathodes driven by green light is approximately $10 \%$ [8,11]. It is very challenging to improve the $\mathrm{QE}$ and maintain the low thermal emittance for this cathode material.

In recent years, surface plasmon polaritons (SPPs) have been used in various fields because of their unique characteristics, such as exceptional absorption and local near field enhancement [12-14]. These features also have the potential to improve the performance of photocathodes. It has been demonstrated that the introduction of SPPs can effectively improve the photoelectron yield of metal photocathodes due to the improved light absorption and local near field enhancement, while the thermal emittance is degraded [15-17].

In this paper, the SPPs were first introduced into the $\mathrm{K}_{2} \mathrm{CsSb}$ photocathode. In order to improve the $\mathrm{QE}$ and obtain low thermal emittance, a nanopatterned $\mathrm{K}_{2} \mathrm{CsSb}$ photocathode was designed to excite the SPPs, enhancing the effective absorption of the incident light and artificially modulating the initial photoelectron distribution in the photocathode. To better understand the effect of SPPs on the $\mathrm{K}_{2} \mathrm{CsSb}$ photocathode performance, Monte Carlo simulations were performed.

The remainder of this paper is organized as follows. The typical Monte Carlo model of photoemission is briefly described in Sec. II A. Based on Monte Carlo simulations, 
the effect of incident light absorption and initial photoelectron distribution on the $\mathrm{K}_{2} \mathrm{CsSb}$ photocathode performance is discussed in Sec. II B. Section III A presents the design of the plasmon-enhanced $\mathrm{K}_{2} \mathrm{CsSb}$ photocathode. The modification of the Monte Carlo model to simulate the photoemission processes of the $\mathrm{K}_{2} \mathrm{CsSb}$ photocathode with SPPs is presented in Sec. III B. The performance of the plasmon-enhanced $\mathrm{K}_{2} \mathrm{CsSb}$ photocathode with different film thicknesses is evaluated and analyzed in Sec. III C. The effect of tuning the incident laser photon energy on the performance of the plasmon-enhanced $\mathrm{K}_{2} \mathrm{CsSb}$ photocathode is elucidated in Sec. IIID. The influence of the plasmonic near field (PNF) induced by SPPs and distortions of the applied accelerating field caused by the nanopattern structure on the thermal emittance is investigated in Sec. III E. A summary is provided in Sec. IV.

\section{MONTE CARLO SIMULATION OF PHOTOEMISSION}

\section{A. Monte Carlo simulation model}

In order to optimize the performance of the plasmonenhanced $\mathrm{K}_{2} \mathrm{CsSb}$ photocathode, Monte Carlo simulations were performed to better understand the photoemission characteristics. The Monte Carlo model is based on Spicer's three-step photoemission model, including the generation of photoelectrons, transport of electrons, and photoelectron emission at the surface [18].

The first step, which involves photon absorption and photoelectron transition from the valence band to the conduction band, determines the initial energy, velocity, and location of each photoelectron. The $\mathrm{K}_{2} \mathrm{CsSb}$ film is deposited on a flat metal substrate. When the laser penetrates the $\mathrm{K}_{2} \mathrm{CsSb}$ film, electrons in the valence band can absorb photons and be excited to the empty state above the conduction band bottom with the energy equal to the sum of the photon energy and initial electron energy. In the simulations, the multiphoton absorption processes were ignored. Without considering the reflections, the laser power exponentially decays along the transmission path due to the absorption, as given by Eq. (1):

$$
I=I_{0} e^{-\alpha(\hbar \omega) z}
$$

where $I_{0}$ is the initial incident laser power and $\alpha(\hbar \omega)$ is the absorption coefficient of $\mathrm{K}_{2} \mathrm{CsSb}$ determining the absorption depth of the incident light relative to the photon energy $\hbar \omega$ [19]. $z$ is a position along the thickness of the $\mathrm{K}_{2} \mathrm{CsSb}$ photocathode. The initial photoelectron distribution, which is also exponential along the $z$ axis, can be obtained by Eq. (1). The position of $y$ is generated randomly and uniformly in the simulations. The initial electron energies are determined by the photon energy of the laser and the density of states (DOS) of $\mathrm{K}_{2} \mathrm{CsSb}$ [20], as given by Eq. (2):

$$
n_{i}=N_{0} \frac{N\left(E_{i}\right) N\left(E_{i}-\hbar \omega\right) \Delta E}{\int_{E_{g}}^{\hbar \omega} N\left(E_{i}-\hbar \omega\right) N\left(E_{i}\right) d E_{i}}
$$

where $N_{0}$ is the initial number of photoelectrons; $N(E)$ is the DOS function of $\mathrm{K}_{2} \mathrm{CsSb} ; E_{g}$ is the band gap of $\mathrm{K}_{2} \mathrm{CsSb} ; E_{i}$ is the electron energy in the conduction band; $n_{i}$ is the number of electrons with energy of $E_{i}$; and $\Delta E$ is the energy interval of the DOS interpolation. The initial velocities of the electrons are given by their energies, and the velocity directions are generated randomly and uniformly in the simulations.

In the second step, the electrons start to diffuse with the initial velocities. Three types of scatterings are considered in this step: electron-electron scattering, electron-hole scattering, and electron-phonon scattering. The mean-free path of the electron-phonon scattering used in the simulation is $3 \mathrm{~nm}$. The mean-free paths of the electron-electron and electron-hole scatterings, which are energy-dependent, are derived from Ref. [21]. The drift step for this process is less than the minimum of the scattering mean-free path of the three scatterings [18]. In the scattering process, the electron holds the possibility in either losing or gaining energy [21], and the law of energy conservation is always satisfied. The electron velocities change with energy, the velocity directions are randomly generated after each scattering event.

When electrons arrive at the photocathode surface, the electrons whose energies exceed the electron affinity can escape from the cathode, emitting into the vacuum. In such a process, the conservation laws of energy and transverse momentum are always satisfied, as presented in Eqs. (3) and (4), respectively:

$$
\begin{gathered}
E^{\mathrm{Out}}=E^{\mathrm{In}}-E_{a}+E_{\mathrm{sch}} \\
P_{\|}^{\mathrm{Out}}=P_{\|}^{\mathrm{In}}
\end{gathered}
$$

where $E^{\text {In }}$ and $E^{\text {Out }}$ are the electron energy values before and after emission, respectively; $E_{a}$ is the electron affinity of photocathode; $E_{\mathrm{sch}}$ is the barrier reduction by the Schottky effect, and $P_{\|}^{\mathrm{In}}$ and $P_{\|}^{\text {Out }}$ are the transverse momentums of the electron before and after emission, respectively. If the energy of the electron arriving at the photocathode surface is lower than the electron affinity, then the electron is reflected back into the photocathode and can continue to scatter with other electrons or phonons. When the energy of each electron remaining inside the cathode is less than one phonon energy, the simulation is terminated. The number of electrons emitted into the vacuum is counted during the simulation. The $\mathrm{QE}$ of the photocathode is given by Eq. (5):

$$
\mathrm{QE}=\frac{N_{\text {vac }}}{N_{\text {tot }}}
$$


TABLE I. Simulation parameters.

\begin{tabular}{lcc}
\hline \hline Parameter & Value & Unit \\
\hline Absorption coefficient & $\alpha(\hbar \omega)=19.82-27.95 \hbar \omega+11.15(\hbar \omega)^{2}$ & $\times 10^{4} \mathrm{~cm}^{-1}$ \\
Photon energy & 2.32 & $\mathrm{eV}$ \\
Number of photons & 300000 & $\mathrm{eV}$ \\
Phonon energy & 0.0027 & $m_{0}:$ the mass of the free electron \\
Average effective mass & $0.1175 m_{0}$ & $\mathrm{eV}$ \\
$E_{\text {sch }}$ & 0 & \\
\hline \hline
\end{tabular}

where $N_{\text {vac }}$ is the number of electrons emitted into the vacuum and $N_{\text {tot }}$ is the total number of incident photons. The emittance of the electron beam emitted from the photocathode is given by Eq. (6):

$$
\frac{\varepsilon_{n}}{\sigma_{y}}=\frac{\sqrt{p_{y}^{2}}}{m c}
$$

where $\frac{\varepsilon_{n}}{\sigma_{y}}$ is the intrinsic emittance of the photocathode (unit: $\mathrm{mm} \mathrm{mrad} / \mathrm{mm}) ; \sqrt{p_{y}^{2}}$ is the root mean square (rms) of the transverse momentum of electrons emitted into the vacuum; $\sigma_{y}$ is the rms of the transverse size of the electron beam; $m$ is the electron mass; and $c$ is the speed of light in vacuum.

\section{B. Analysis of photoemission process}

Based on the Monte Carlo code, the photoelectron emission characteristics are studied. The parameters used in the simulation are listed in Table I. The photocathode is illuminated by the light of $532 \mathrm{~nm}$ in the simulations. As shown in Fig. 1(a), the number of initial photoelectrons

(a)

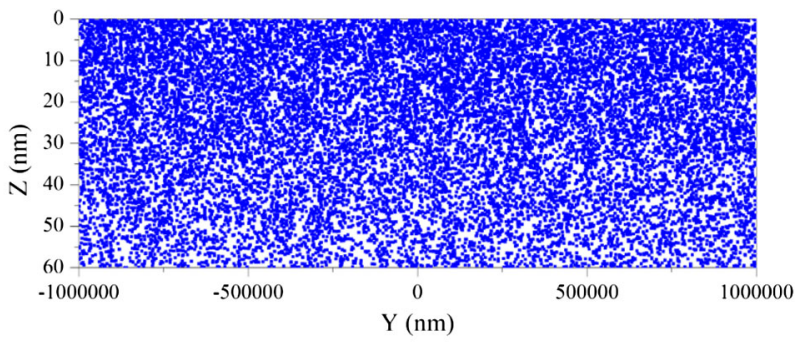

(b)

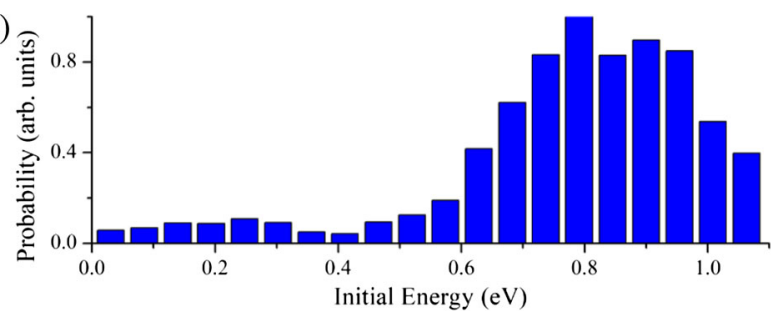

FIG. 1. Simulation results of initial photoelectron characteristics of $\mathrm{K}_{2} \mathrm{CsSb}$ photocathode: (a) Initial position distribution (photocathode surface is at $z=0$, and photocathode thickness is $60 \mathrm{~nm}$ ); (b) initial energy distribution (incident photon energy: $2.32 \mathrm{eV})$. generated in the $\mathrm{K}_{2} \mathrm{CsSb}$ photocathode decays exponentially along the $z$ axis. Figure 1(b) shows the initial energy distribution of photoelectrons generated in the $\mathrm{K}_{2} \mathrm{CsSb}$ photocathode.

Figure 2 presents the simulation results of the $\mathrm{QE}$ and intrinsic emittance of the $\mathrm{K}_{2} \mathrm{CsSb}$ photocathodes with different thicknesses. The results indicate that the $\mathrm{QE}$ increases with the $\mathrm{K}_{2} \mathrm{CsSb}$ photocathode thickness and approaches saturation at approximately $20 \mathrm{~nm}$. The intrinsic emittance decreases with the film thickness of the $\mathrm{K}_{2} \mathrm{CsSb}$ photocathode and is close to the constant value of $0.27 \mathrm{~mm} \mathrm{mrad} / \mathrm{mm}$ when the thickness exceeds $20 \mathrm{~nm}$. Compared with the simulation results of the QE, the intrinsic emittance simulation is more sensitive to the effect of scatterings with the limited number of initial photoelectrons used in the simulation. The intrinsic emittance curve exhibits an oscillating behavior along the photocathode thickness.

The initial photoelectron distribution is determined by the absorption of the incident light in the cathode conforming to the law of exponential decay. The absorption depth $(1 / \mathrm{e})$ of $\mathrm{K}_{2} \mathrm{CsSb}$ with the photon energy of $2.32 \mathrm{eV}$ is approximately $66.7 \mathrm{~nm}$. As shown in Fig. 3, with a thickness of $20 \mathrm{~nm}$, the absorbed power of the incident light is just about $30 \%$ of the total power. The photons absorbed at deeper positions make little contribution to the

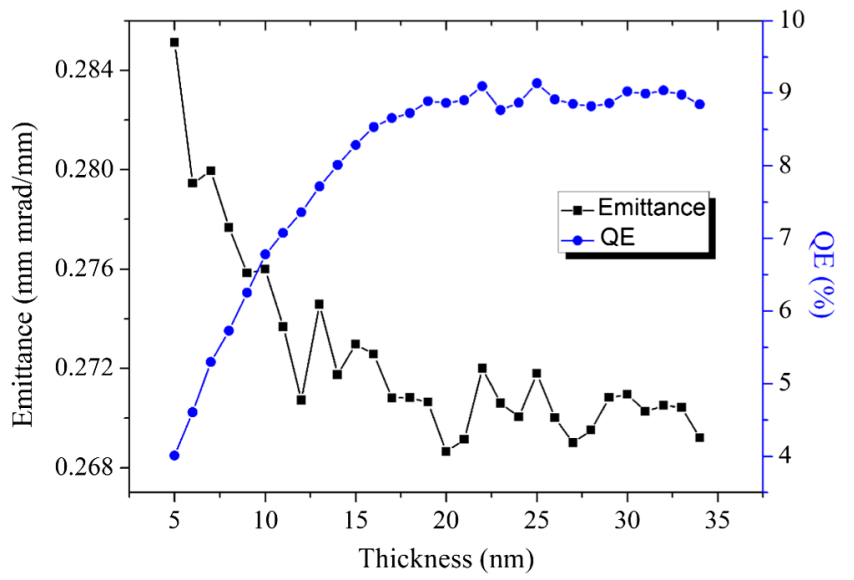

FIG. 2. Simulation results of $\mathrm{QE}$ and intrinsic emittance of $\mathrm{K}_{2} \mathrm{CsSb}$ photocathode with different thicknesses; blue and black lines indicate QE and intrinsic emittance, respectively. 


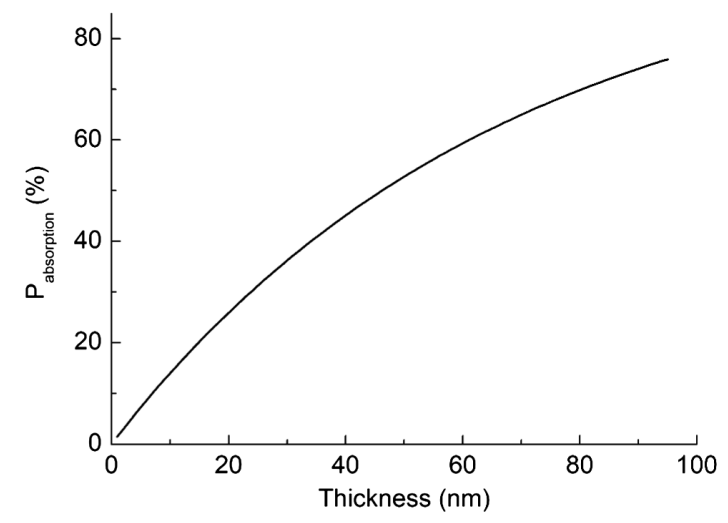

FIG. 3. Light absorption with the depth of cathode.

photoelectron emission. Most of the incident laser power is not used for the typical $\mathrm{K}_{2} \mathrm{CsSb}$ photocathode.

Figure 2 shows that the relationship between QE, intrinsic emittance, and photocathode thickness is nonlinear. In addition to the exponentially decaying absorption of incident light, it may also be caused by the different contributions of photoelectrons excited at different depths according to Spicer's three-step photoemission model. To better understand the effect of the initial distribution of photoelectrons on the photocathode performance, the contributions of photoelectrons at different depths were evaluated in this part. The photocathode was sliced along the depth. It was assumed that in each simulation, the photoelectrons produced in each of the slices had the same initial energy distribution, and the transport and emission mechanisms of the photoelectrons were unchanged as the model described above. The number of photoelectrons produced in each slice was 300000 .

The blue line in Fig. 4 indicates the emission probability of the photoelectrons from the slices at different depths.

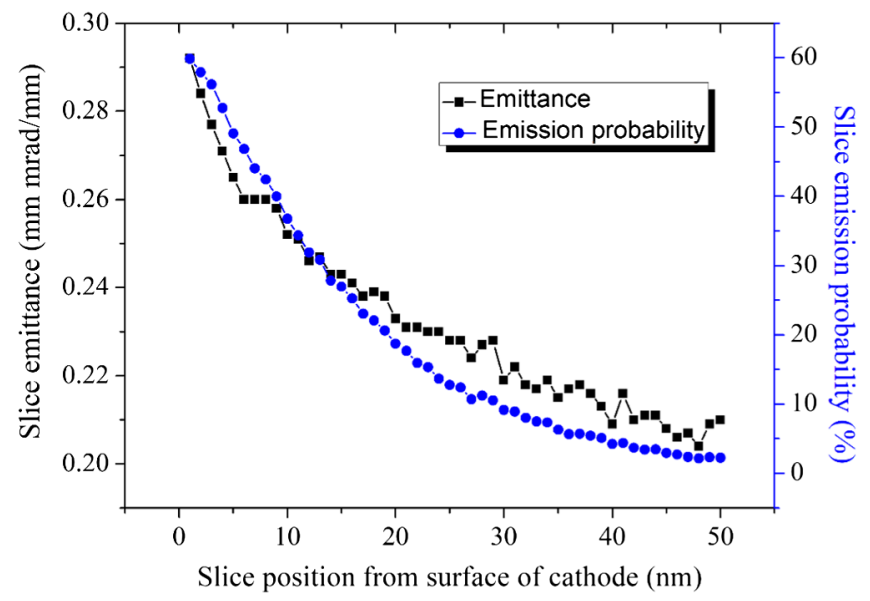

FIG. 4. Simulation results of $\mathrm{K}_{2} \mathrm{CsSb}$ photocathode performance when all initial photoelectrons are generated in one slice. The blue line indicates the emission probability. The black line represents the intrinsic emittance. (The thickness of the slice is $1 \mathrm{~nm}$.)
The emission probability is defined as the ratio of the number of photoelectrons emitted into the vacuum to that produced in each slice. The black line represents the results of the intrinsic emittance of the photocathode when the initial photoelectrons are produced in one slice. The scattering probability increases with the transport distance, and the emission probability and transverse momenta of the photoelectrons decrease with the photocathode depth. The contribution of photoelectrons to QE and intrinsic emittance decreases with increasing depth. As the QE and intrinsic emittance of the photocathode are the statistical results of the total contribution of the initial photoelectrons, it is worth mentioning that they can be improved by adjusting the initial distribution of the photoelectron along the depth direction.

\section{PLASMON-ENHANCED BIALKALI PHOTOCATHODE}

Based on the previous analysis, the maximum efficiency of the typical $\mathrm{K}_{2} \mathrm{CsSb}$ film photocathode in absorbing the incident laser is approximately $30 \%$. The exponential decay distribution of the initial photoelectron density determined by the optical absorption properties of the photocathode is not conducive to achieving the best performance. In order to improve the QE and control the emittance, the SPPs are introduced into the $\mathrm{K}_{2} \mathrm{CsSb}$ photocathode to enhance the absorption of the incident laser and artificially regulate the initial photoelectron distribution in the photocathode.

\section{A. Photocathode design}

To protect the photoemission properties of the surface, a silver substrate with a nanopattern is employed to achieve wave vector matching, exciting the SPPs at the interface between the substrate and $\mathrm{K}_{2} \mathrm{CsSb}$ photocathode. Figure 5 presents the structural diagram of the photocathode. To simplify the simulation, a corrugated nanopattern is designed on the surface of the substrate. The $\mathrm{K}_{2} \mathrm{CsSb}$ film is deposited with a certain thickness on the nanopatterned silver substrate.

The geometrical parameters are optimized by an electromagnetic field simulation software (CST Studio) [22] to ensure that the SPPs can be successfully excited at a specific wavelength. Figure 6 shows the simulation results

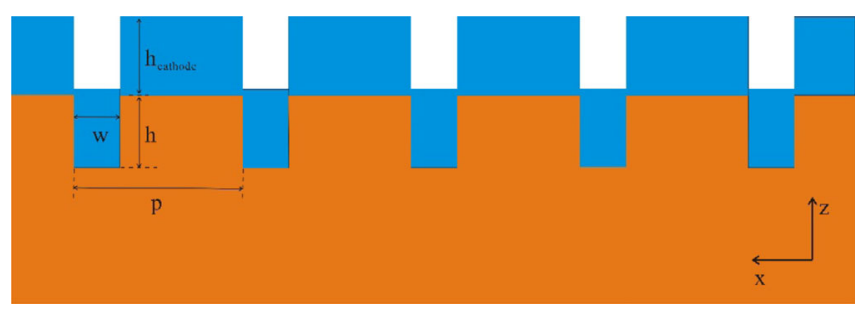

FIG. 5. Diagram of photocathode cross section with nanopattern. (The blue represents $\mathrm{K}_{2} \mathrm{CsSb}$ film, the yellow represents silver substrate.) 


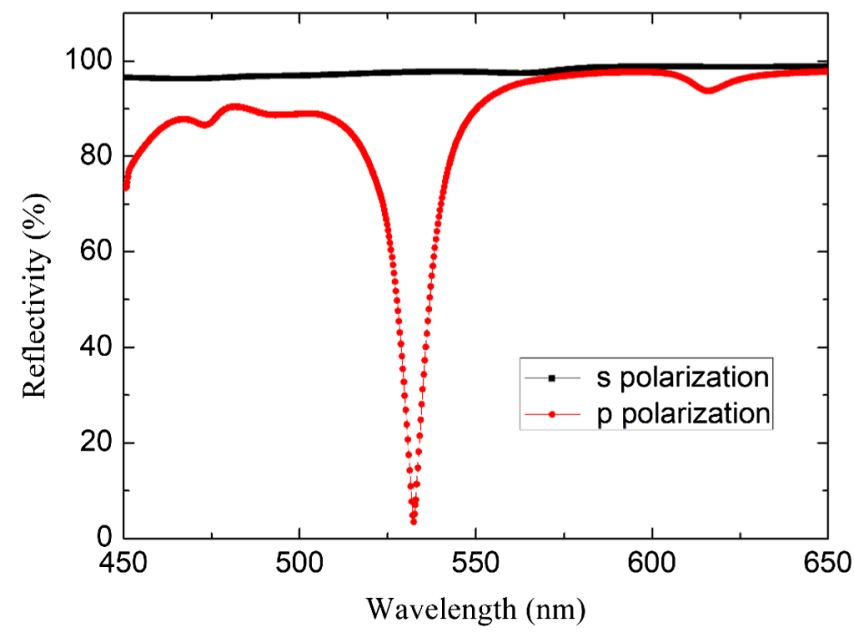

FIG. 6. Simulated reflectivity spectrum of photocathode whose geometrical parameters are $p=505 \mathrm{~nm}, h=84 \mathrm{~nm}, w=88 \mathrm{~nm}$, and $h_{\text {cathode }}=16 \mathrm{~nm}$.

of the reflectivity spectrum of the photocathode whose geometrical parameters of $p=505 \mathrm{~nm}, h=84 \mathrm{~nm}$, $w=88 \mathrm{~nm}$, and $h_{\text {cathode }}=16 \mathrm{~nm}$. In the simulation, the incident light is perpendicularly irradiated on the photocathode surface. The red and black lines indicate the reflectivity spectra of the photocathode with the incident lights of $p$ and $s$ polarizations, respectively. When the photocathode is irradiated by the incident laser of $p$ polarization, the exceptionally enhanced absorption close to $95 \%$ occurs at the wavelength of $532 \mathrm{~nm}$, which indicates that the SPPs are successfully excited.

When the SPPs are excited, the electromagnetic field near the interface between the $\mathrm{Ag}$ substrate and $\mathrm{K}_{2} \mathrm{CsSb}$ film is redistributed. The distribution of the PNF is periodic and aligned with the nanopattern structure. Figure 7 shows the simulation results of the two-unit cells with a phase of $90^{\circ}$; the color contour represents the normalized electric field strength in relative units. The lines outline the two-unit cell of the nanopattern structure. It indicates that the electromagnetic field near the interface is enhanced in the vicinity of the interface between the $\mathrm{Ag}$ substrate and $\mathrm{K}_{2} \mathrm{CsSb}$ film.

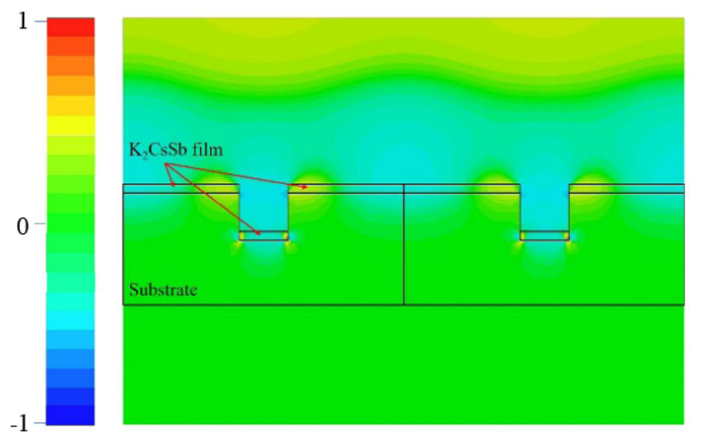

The PNF distribution can be regulated by adjusting the geometrical parameters of the nanopattern structure. With the introduction of SPPs, the significantly reduced reflectivity and adjustable PNF provide the possibility of improving the $\mathrm{K}_{2} \mathrm{CsSb}$ photocathode performance.

\section{B. Monte Carlo model with SPPs}

In order to demonstrate the effect of SPPs on the photocathode performance, the Monte Carlo simulation model described above is modified. When the SPPs are excited, the absorption of the incident light increases, and the energy density distribution in the electromagnetic field changes. Based on Spicer's three-step photoemission model, the introduction of SPPs influences the generation of photoelectrons. When the incident laser is irradiated on the photocathode, it passes through the $\mathrm{K}_{2} \mathrm{CsSb}$ film and decays exponentially. When it arrives at the interface between the $\mathrm{K}_{2} \mathrm{CsSb}$ film and nanopatterned substrate, it excites the SPPs. With the geometrical parameter optimization of the nanopattern structure, the reflection at the interface between the $\mathrm{K}_{2} \mathrm{CsSb}$ film and nanopatterned substrate is reduced to extremely low levels. The reflection at this interface can be ignored in the simulations; the incident photon energy is converted into plasmonic energy at the interface. The total plasmonic energy is eventually absorbed by the $\mathrm{K}_{2} \mathrm{CsSb}$ film and metal substrate. The light energy absorbed by the $\mathrm{K}_{2} \mathrm{CsSb}$ film consists of two parts. One part is absorbed during the process in which light passes through the $\mathrm{K}_{2} \mathrm{CsSb}$ film and can be obtained using Eq. (1); the other part is obtained from the SPPs.

The distribution of the photoelectrons, which are generated from the transmission process, is the same as that described above. Its quantity is given by the following:

$$
N_{t}=N_{p}\left(1-e^{-\alpha(\hbar \omega) h_{\text {cathode }}}\right)
$$

where $N_{t}$ is the total number of photoelectrons generated from the transmission process and $N_{p}$ is the total number of photons irradiated on the photocathode. When the incident light arrives at the interface between the $\mathrm{K}_{2} \mathrm{CsSb}$ film and nanopatterned substrate and excites the SPPs, the enhanced

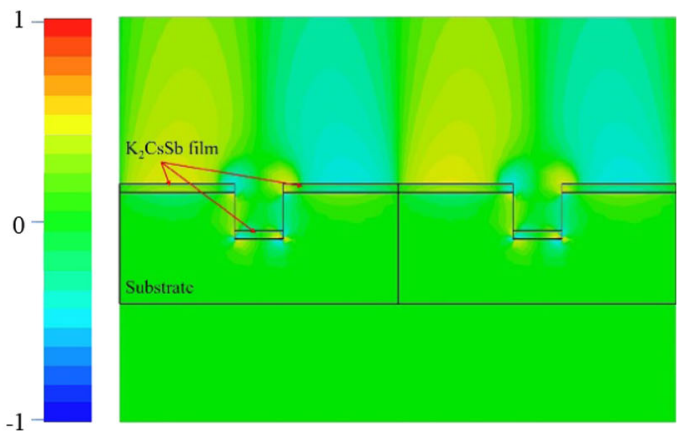

FIG. 7. Electric field distribution of PNF over cross section. Left and right images show electric field distributions of $X$ and $Y$ components of PNF, respectively. 
PNF occurs. Based on the law of conservation of energy and assuming that there is no reflection at the interface after the optimization of the geometrical parameters of the photocathode, the plasmonic energy is eventually absorbed by the metal substrate and $\mathrm{K}_{2} \mathrm{CsSb}$ film. To simplify the simulation model, it is assumed that the total amount of plasmonic energy absorbed by the $\mathrm{K}_{2} \mathrm{CsSb}$ film is used to generate the photoelectrons. The nonlinear photoemissions are not considered owing to the photon energy of the incident light $[15,16]$. In fact, hot electrons can also be generated in the silver substrate [14]. The energy of all hot electrons generated in the silver substrate is assumed to be finally converted to heat by electron-electron and electron-phonon scatterings. The initial distribution of photoelectrons from the SPPs is determined by the energy distribution of the PNF in the $\mathrm{K}_{2} \mathrm{CsSb}$ film. Its quantity $N_{\text {spp_semi }}$ can be obtained by the following:

$$
N_{\text {spp_semi }}=\left(N_{p}-N_{t}\right) \times \frac{E_{\text {spp_semi }}}{E_{\text {spp_total }}}
$$

where $E_{\text {spp_semi }}$ is the energy of the PNF in the $\mathrm{K}_{2} \mathrm{CsSb}$ film and $E_{\text {spp_total }}$ is the total energy of the PNF. The $E_{\text {spp_semi }}$ and the $E_{\text {spp_total }}$ values can be obtained from the simulation results using CST Studio. The total number of photoelectrons generated in the $\mathrm{K}_{2} \mathrm{CsSb}$ film can be calculated as the sum of $N_{t}$ and $N_{\text {spp_emi }}$. The initial energy of the photoelectrons is given by Eq. (2).

Without considering the effect of external fields (e.g., accelerating field and PNF) on the movements of photoelectrons, the influence of SPPs on the transport step is negligible because their introduction does not change the electronic properties of the $\mathrm{K}_{2} \mathrm{CsSb}$ film. To ensure that the photoemission properties of the $\mathrm{K}_{2} \mathrm{CsSb}$ photocathode surface are not changed, the nanopattern structure used to excite the SPPs is fabricated on the silver substrate. It can be considered that the SPPs also have no influence on the step of emission on the surface.

\section{Simulation and analysis of photoemission process with SPPs}

In order to simulate the photoemission process of the photocathode with SPPs, the geometrical parameters of the nanopattern on the surface of the substrate are optimized using CST Studio to ensure that the SPPs can be excited at the wavelength of $532 \mathrm{~nm}$. The reflectivity of the photocathode needs to be reduced to a certain value. The PNF distribution can be given using CST Studio after the excitation of SPPs. Using the obtained PNF results, the performance of the photocathode with SPPs can be explored using the modified Monte Carlo simulation code.

Table II presents the performance of the photocathodes with different $\mathrm{K}_{2} \mathrm{CsSb}$ film thicknesses. The geometrical parameters (i.e., $p, w$, and $h$ ) of the aforementioned photocathodes are obtained through optimization to ensure that the SPPs are successfully excited, and the reflectivity of the photocathode is $<5 \%$. Without the SPPs, the incident light absorption values of the $\mathrm{K}_{2} \mathrm{CsSb}$ photocathodes with thicknesses of $14,16,18$, and $20 \mathrm{~nm}$ are $18.92 \%, 21.40 \%, 23.67 \%$, and $25.97 \%$, respectively. Based on the Monte Carlo simulation, the QEs of these photocathodes are $5.68 \%, 6.54 \%, 7.37 \%$, and $8.22 \%$, respectively. With the introduction of SPPs, the reflectivity of the incident light is reduced to approximately $\sim 5 \%$ at a wavelength of $532 \mathrm{~nm}$ by optimizing the geometrical parameters of the substrates. The increased incident light absorption by the SPPs is approximately twice as much as that in the transmission process. Figure 8 shows the simulation results of the initial photoelectron density distribution in the $\mathrm{K}_{2} \mathrm{CsSb}$ photocathode with SPPs. The orange part in the figure is one element of the nanopatterned substrate. The geometrical parameters of the substrate are listed in Table II. The 20-nm $\mathrm{K}_{2} \mathrm{CsSb}$ film is deposited onto the surface of the substrate. The density of the blue particles represents the initial photoelectron density in the $\mathrm{K}_{2} \mathrm{CsSb}$ film. Due to the increased incident light absorption by the SPPs, the initial photoelectron distribution in the photocathode is regulated and ceases

TABLE II. Photocathode performance with SPPs.

\begin{tabular}{lcccc}
\hline \hline Thickness $h_{\text {cathode }}(\mathrm{nm})$ & 14 & 16 & 18 & 20 \\
\hline$p(\mathrm{~nm})$ & 530 & 505 & 480 & 450 \\
$w(\mathrm{~nm})$ & 90 & 88 & 101 & 112 \\
$h(\mathrm{~nm})$ & 89 & 84 & 98 & 103 \\
Reflectivity $(\%)$ & 3 & 5 & 3 & 5 \\
QE $\%)$ & 17.33 & 19.40 & 17.15 & 24.49 \\
Emittance $(\mathrm{mm} \mathrm{mrad} / \mathrm{mm})$ & 0.282 & 0.279 & 0.276 & 0.276 \\
QE $(\%)$ without SPPs & 6.67 & 7.42 & 8.19 & 8.84 \\
Emittance without SPPs $(\mathrm{mm} \mathrm{mrad} / \mathrm{mm})$ & 0.281 & 0.276 & 0.277 & 0.273 \\
Normal absorption $(\%)$ & 18.90 & 21.24 & 23.51 & 26.01 \\
Absorption by SPPs $(\%)$ & 37.29 & 48.41 & 38.52 & 54.42 \\
Total absorption of light $(\%)$ & 56.19 & 69.65 & 62.02 & 80.44 \\
\hline \hline
\end{tabular}




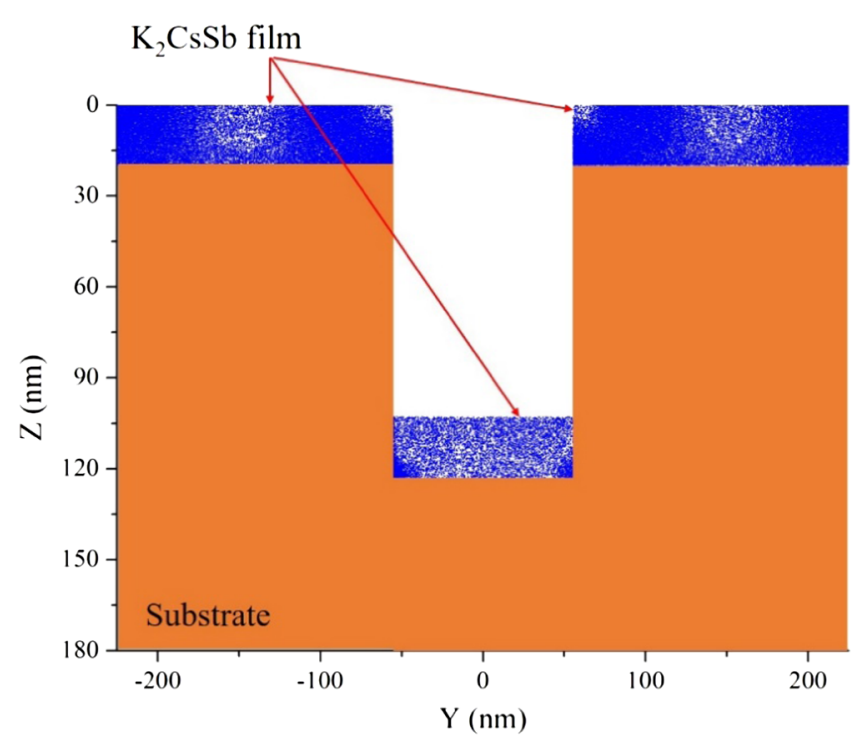

FIG. 8. Simulation result of initial photoelectron density distribution in $\mathrm{K}_{2} \mathrm{CsSb}$ photocathode with SPPs $\left(\mathrm{K}_{2} \mathrm{CsSb}\right.$ film thickness: $20 \mathrm{~nm}$ ).

to decay exponentially perpendicular to the surface of the $\mathrm{K}_{2} \mathrm{CsSb}$ film.

With the SPPs, the QEs of the photocathode with thicknesses of 14, 16, 18, and $20 \mathrm{~nm}$ are approximately $17.33 \%, 19.40 \%, 17.15 \%$, and $24.49 \%$, respectively. Because of the increased number of photoelectrons in the deep layers of the $\mathrm{K}_{2} \mathrm{CsSb}$ film, the tripled absorption of incident light approximately doubles the QE, as illustrated in Fig. 6. The intrinsic emittances of the photocathode with or without the SPPs are almost unchanged due to the modulated photoelectron distribution, which holds great promise for breaking the trade-off between the $\mathrm{QE}$ and intrinsic emittance of the photocathode. Moreover, it is worth noting that the 18 -nm-thick photocathode has the lowest $\mathrm{QE}$ among the photocathodes. This is because the geometric parameters $(p, w$, and $h)$ of the photocathodes with different thicknesses are optimized to ensure that the SPPs are successfully excited, and the reflectivity of photocathodes is $<5 \%$. As shown in Fig. 9, although the

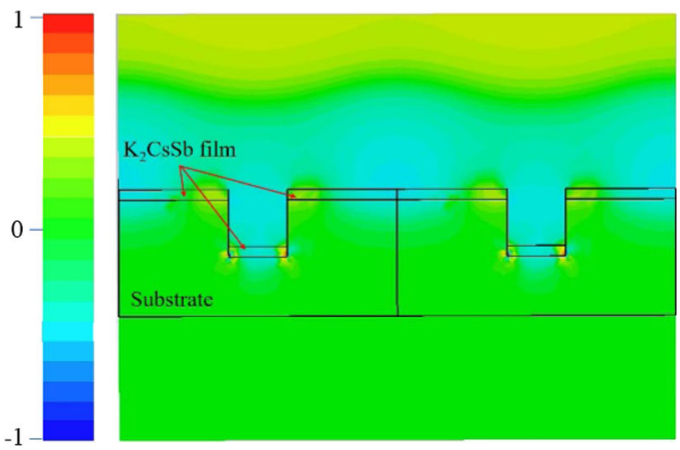

target reflectivity is achieved, the distributions of the plasmonic fields for different photocathode thicknesses may differ, resulting in the variation of the light energy absorbed by the $\mathrm{K}_{2} \mathrm{CsSb}$ film from the SPPs.

\section{Photon energy scan}

It is assumed that adjusting the wavelength of the incident light over a narrow range by fine-tuning the geometrical parameters of the nanopattern slightly affects the PNF characteristics and photocathode reflectivity. The calculated PNF with the incident light of $532 \mathrm{~nm}$ can be used to evaluate the effect of the photon energy of the incident light on the QE and intrinsic emittance of the photocathode. Figure 10 presents the simulation results of the relationship between the $\mathrm{QE}$ and photon energy of the incident light. The photocathode with SPPs (black curve) still has higher QEs when the incident photon energy is adjusted over a narrow range (i.e., 2.0-2.5 eV). The absorption coefficient of $\mathrm{K}_{2} \mathrm{CsSb}$ decreases with the incident photon energy. The number of incident photons absorbed in the $\mathrm{K}_{2} \mathrm{CsSb}$ film decreases, leaving more incident photons to excite the SPPs. The ratio of the QE of photocathodes with SPPs to that of photocathodes without SPPs increases with the decrease in the incident photon energy. Figure 11 shows the simulation results of the relationship between the intrinsic emittance of the photocathode and incident photon energy. The intrinsic emittances of the photocathodes with and without SPPs are almost the same with the small range adjustment of the incident photon energy. Comparing Figs. 10 and 11, it can be concluded that the introduction of SPPs allows for a more adjustable range of incident light photon energy to achieve a photocathode with low intrinsic emittance and high QE.

\section{E. Potential risk factors induced by SPPs}

It needs to be mentioned that the introduction of SPPs also brings some potential risk factors that may cause the degradation of the overall emittance of the electron beam emitted from the cathode, such as the introduction of PNF and distortions of the applied accelerating field caused by the nanopattern structure. The influence of PNF on the

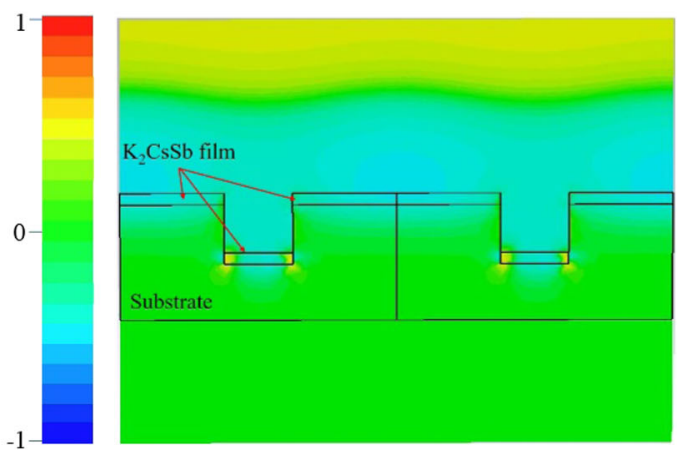

FIG. 9. Electric field distributions of PNF over cross section. Left and right images show electric field distributions of PNFs of 18- and 20-nm photocathodes, respectively. 


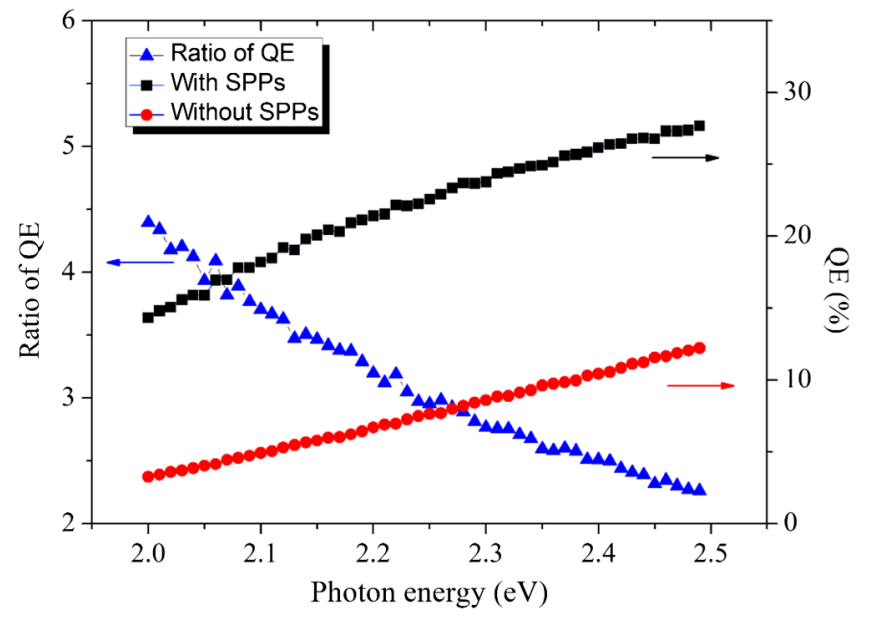

FIG. 10. Simulation results of relationship between QE of photocathode and incident photon energy with/without SPPs. Black and red curves are QEs of photocathodes with and without SPPs, respectively; blue curve represents ratio of QE of photocathodes with SPPs to that of photocathodes without SPPs.

electron beam dynamics has been researched in Ref. [23], which indicates that the PNF can degrade the emittance of the electron beam emitted from the cathode. As the intensity of the PNF exponentially decays perpendicular to the interface, the region of the PNF is limited. The degradation of the emittance can be suppressed by boosting the launching field at the cathode surface to reduce the flight time of the electron beam in this area. Moreover, the intensity of the PNF is related to the power density of incident light; hence, the degradation of the emittance caused by the PNF can also be suppressed by reducing the power density of incident light. Compared with the metal photocathode, the $\mathrm{K}_{2} \mathrm{CsSb}$ photocathode with a higher QE significantly reduces the power density requirement of

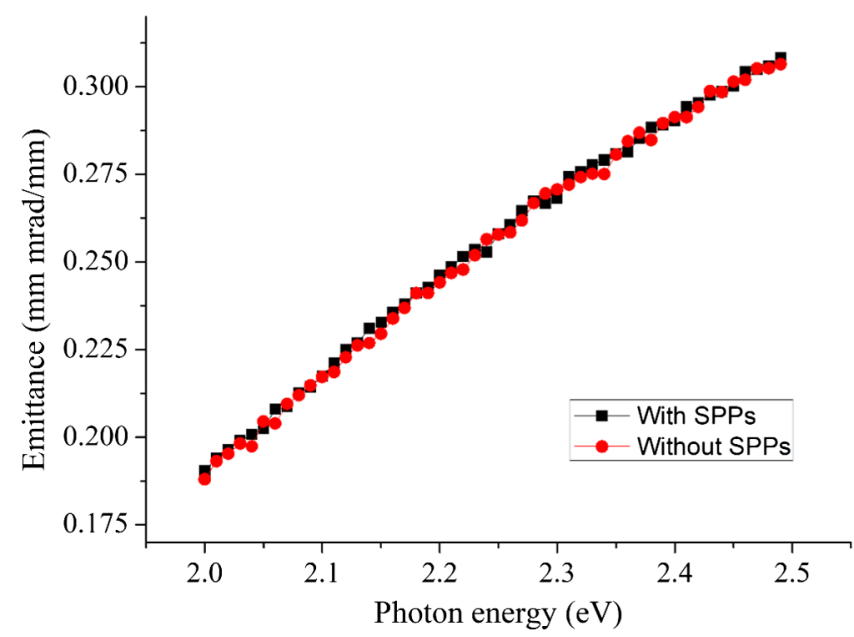

FIG. 11. Simulation results of relationship between intrinsic emittance of photocathode and incident photon energy with/ without SPPs. incident light, which also reduces the influence of the PNF on the electron beam. For example, assuming that the QE of the photocathode is 10\%, the FWHM of the incident light is $10 \mathrm{ps}$, the beam diameter is $2 \mathrm{~mm}$, the required peak power density of the incident light to generate a $100-\mathrm{pC}$ electron beam is $\sim 7.4 \times 10^{-6} \mathrm{GW} / \mathrm{cm}^{2}$. Under these conditions, the emittance growth factor $\left(\varepsilon_{\text {with PNF }} / \varepsilon_{\text {without PNF }}\right)$ caused by the PNF at the launching field of $30 \mathrm{MV} / \mathrm{m}$ can be estimated to be $\sim 1.004$.

The typical emittance growth caused by the roughness includes two types: emittance growth due to the emission angle diffusion and emittance growth induced by the field distortion of the applied electric field caused by the rough surface [24-26]. The photoelectrons emitted from the substrate can be ignored because its photoelectron yield is lower compared with that of the $\mathrm{K}_{2} \mathrm{SbCs}$ photocathode. The thickness of the $\mathrm{K}_{2} \mathrm{SbCs}$ film is far smaller than the diameter of the cathode. Almost all photoelectrons are emitted from the top surface of the $\mathrm{K}_{2} \mathrm{SbCs}$ film. The type of emission angle diffusion has no substantial contribution to the emittance growth for this configuration of the $\mathrm{K}_{2} \mathrm{SbCs}$ photocathode with SPPs. As shown in Fig. 12, the element of the groove distorts the applied electric field near the groove. It locally acts as a focusing lens for the photoelectrons emitted from the bottom of the groove, which is similar to the effect of the grid mesh on the electron beam in a thermionic gridded electron gun [27]. The beam emittance due to the groove array is proportional to the width of the groove and radius of the light spot and depends on the launching field. The width of the groove adopted in the photocathode is in the order of tens of nanometers; this is substantially smaller than the typical mesh hole size (tens of micrometers) used in the thermionic gridded electron gun. Hence, the emittance growth caused by field distortions is also small. For the photocathode whose geometrical parameters are $p=450 \mathrm{~nm}, h=$ $103 \mathrm{~nm}, w=112 \mathrm{~nm}$, and $h_{\text {cathode }}=20 \mathrm{~nm}$, when the

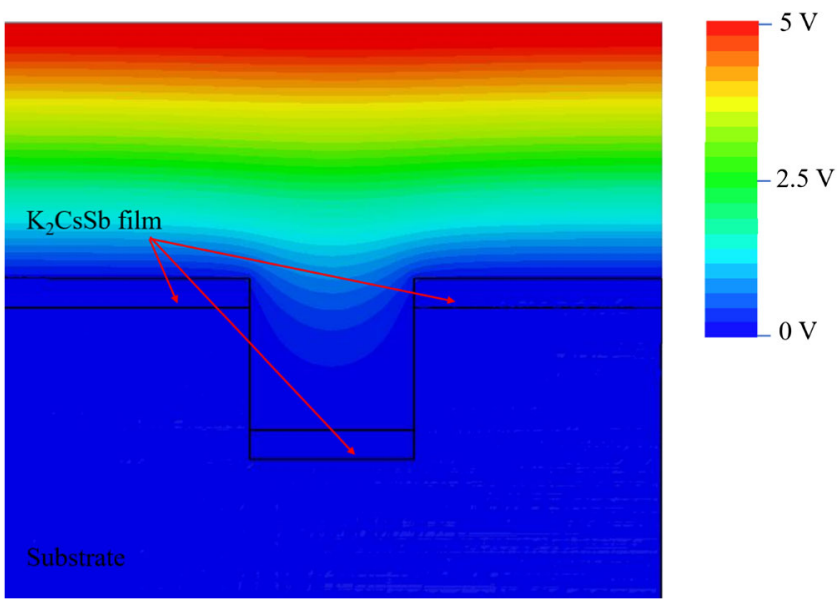

FIG. 12. Potential distribution of one unit cell of nanopattern at cross section with launching field of $30 \mathrm{MV} / \mathrm{m}$. 
diameter of the light spot is $2 \mathrm{~mm}$, the emittance caused by field distortions under a launching field of $30 \mathrm{MV} / \mathrm{m}$ can be estimated to be $\sim 5.6 \times 10^{-3} \mathrm{~mm} \mathrm{mrad} / \mathrm{mm}$ using Eq. (38) of Ref. [27], which is considerably lower than the intrinsic emittance of the photocathode. Moreover, as the SPPs can be excited by different nanopatterns, it provides the flexibility to reduce the emittance degradation caused by the nanopattern by optimizing the geometrical structure.

\section{CONCLUSION}

In conclusion, the photoemission simulations of the $\mathrm{K}_{2} \mathrm{CsSb}$ photocathode indicate that the effective absorption of incident light is approximately $30 \%$ of the total power. The initial distribution of the photoelectrons plays an important role in the performance of photocathodes due to the different contributions of the photoelectrons at different depths. Based on these characteristics, the SPPs are introduced by the groove nanopattern to improve the effective absorption of incident light and modulate the initial photoelectron distribution. The Monte Carlo photoemission model of the $\mathrm{K}_{2} \mathrm{CsSb}$ photocathode with SPPs was first established and used to study the effect of the introduction of SPPs. The simulation results show that the QE of the $\mathrm{K}_{2} \mathrm{CsSb}$ photocathode with SPPs is 2-3 times that of the photocathode without SPPs. Furthermore, the thermal emittance of the photocathode remains substantially unchanged, which breaks the trade-off between the photocathode QE and emittance. Hence, it plays a very important role in the development of high-brightness electron sources.

\section{ACKNOWLEDGMENTS}

The work is partly funded by the National Natural Science Foundation of China under Grant No. 11905276 and Science and Technology Commission of Shanghai Municipality.

[1] W. Ackermann, G. Asova, V. Ayvazyan et al., Operation of a free-electron laser from the extreme ultraviolet to the water window, Nat. Photonics 1, 336 (2007).

[2] W. S. Graves, F. X. Kartner, D. E. Moncton, and P. Piot, Intense Superradiant $X$ Rays from a Compact Source Using a Nanocathode Array and Emittance Exchange, Phys. Rev. Lett. 108, 263904 (2012).

[3] N. D. Browning, M. A. Bonds, G. H. Campbell, J.E. Evans, T. LaGrange, K. L. Jungjohann, D. J. Masiel, J. McKeown, S. Mehraeen, B. W. Reed, and M. Santala, Recent developments in dynamic transmission electron microscopy, Curr. Opin. Solid State Mater. Sci. 16, 23 (2012).

[4] D. H. Dowell, I. Bazarov, B. Dunham, K. Harkay, C. Hernandez-Garcia, R. Legg, H. Padmore, T. Rao, J.
Semdley, and W. Wan, Cathode R\&D for future light sources, Nucl. Instrum. Methods Phys. Res., Sect. A 622, 685 (2010).

[5] Z. Ding and S. Karkare, J. Feng, D. Filippetto, M. Johnson, S. Virostek, F. Sannibale, J. Nasiatka, M. Gaowei, J. Sinsheimer, E. Muller, J. Smedley, and H. Padmore, Temperature-dependent quantum efficiency degradation of $\mathrm{K}-\mathrm{Cs}-\mathrm{Sb}$ bialkali antimonide photocathodes grown by a triple-element codeposition method, Phys. Rev. Accel. Beams 20, 113401 (2017).

[6] J. Maxson, L. Cultrera, C. Gulliford, and I. Bazarov, Measurement of the tradeoff between intrinsic emittance and quantum efficiency from a NaKSb photocahode near threshold, Appl. Phys. Lett. 106, 234102 (2015).

[7] D. H. Dowell and J. F. Schmerge, Quantum efficiency and thermal emittance of metal photocathodes, Phys. Rev. ST Accel. Beams 12, 074201 (2009).

[8] M. A. H. Schmeißer, S. Mistry, H. Kirschner, S. Schubert, A. Jankowiak, T. Kamps, and J. Kühn, Towards the operation of Cs-K-Sb photocathodes in superconducting rf photoinjectors, Phys. Rev. Accel. Beams 21, 113401 (2018).

[9] I. A. Dubovoi, A. S. Chernikov, A. M. Prokhorov, M. Ya. Schelev, and V. N. Ushakov, Multialkali photocathodes grown by molecular beam epitaxy technique, in Proceedings of the 9th International Congress on High-Speed Photography and Photonics (1991), p. 1358, https:// doi.org/10.1117/12.24018.

[10] M. Gaowei, Z. Ding, S. Schubert, H. B. Bhandari, J. Sinsheimer, J. Kuehn, V. V. Nagarkar, M. S. J. Marshall, J. Walsh, E. M. Muller, K. Attenkofer, H. J. Frisch, H. Padmore, and J. Smedley, Synthesis and X-ray characterization of sputtered bi-alkali antimonide photocathodes, APL Mater. 5, 116104 (2017).

[11] M. A. Mamun, C. Hernandez-Garcia, M. Poelker, and A. A. Elmustata, Correlation of $\mathrm{CsK}_{2} \mathrm{Sb}$ photocathode lifetime with antimony thickness, APL Mater. 3, 066103 (2015).

[12] W. L. Barnes, A. Dereux, and T. W. Ebbesen, Surface plasmon subwavelength optics, Nature (London) 424, 824 (2003).

[13] S. Linic, P. Christopher, and D. B. Ingram, Plasmonicmetal nanostructures for efficient conversion of solar to chemical energy, Nat. Mater. 10, 911 (2011).

[14] C. Clavero, Plasmon-induced hot-electron generation at nanoparticle/metal-oxide interfaces for photovoltaic and photocatalytic devices, Nat. Photonics 8, 95 (2014).

[15] A. Polyakov, C. Senft, K. F. Thompson, J. Feng, S. Cabrini, P. J. Schuck, and H. A. Padmore, Plasmon-Enhanced Photocathode for High Brightness and High Repetition Rate X-Ray Sources, Phys. Rev. Lett. 110, 076802 (2013).

[16] R. K. Li, H. To, G. Andonian, J. Feng, A. Polyakov, C. M. Scoby, K. Thompson, W. Wan, H. A. Padmore, and P. Musumeci, Surface-Plasmon Resonance-Enhanced Multiphoton Emission of High-Brightness Electron Beams from a Nanostructured Copper Cathode, Phys. Rev. Lett. 110, 074801 (2013).

[17] S. Vilayurganapathy, M. I. Nandasiri, A. G. Joly, P. Z. El-Khoury, T. Varga, G. Coffey, B. Schwenzer, A. Pandey, 
A. Kayani, W. P. Hess, and S. Thevuthasan, Silver nanorod arrays for photocathode applications, Appl. Phys. Lett. 103, 161112 (2013).

[18] H. Xie, I. Ben-zvi, T. Rao, T. Xin, and E. Wang, Experimental measurements and theoretical model of the cryogenic performance of bialkali photocathode and characterization with Monte Carlo simulation, Phys. Rev. Accel. Beams 19, 103401 (2016).

[19] L. Kalarasse, B. Ã. Bennecer, and F. Kalarasse, Optical properties of the alkali antimonide semiconductors $\mathrm{Cs}_{3} \mathrm{Sb}$, $\mathrm{Cs}_{2} \mathrm{KSb}, \mathrm{CsK}_{2} \mathrm{Sb}$ and $\mathrm{K}_{3} \mathrm{Sb}$, J. Phys. Chem. Solids 71, 314 (2010).

[20] A. R. H. F. Ettema and R. A. de Groot, Electronic structure of $\mathrm{Cs}_{2} \mathrm{KSb}$ and $\mathrm{K}_{2} \mathrm{CsSb}$, Phys. Rev. B 66, 115102 (2002).

[21] W. E. Spicer, SLAC Report No. SLAC-PUB-6306, 1993.

[22] https://www.3ds.com/products-services/simulia/products/ cst-studio-suite/.
[23] Z. Jiang, X. Li, D. Huang, M. Zhang, and Q. Gu, Effect of plasmonic near field on the emittance of plasmon-enhanced photocathode, Nucl. Instrum. Methods Phys. Res., Sect. A 897, 14 (2018).

[24] X. He, C. Tang, W. Huang, and Y. Lin, Researches on thermal emittance of metal cathode in photoinjectors, High Energy Phys. Nucl. Phys. 28, 1007 (2004).

[25] Z. Zhang and C. Tang, Analytical study on emittance growth caused by roughness of a metallic photocathode, Phys. Rev. Accel. Beams 18, 053401 (2015).

[26] M. Krasilnikov, Impact of the cathode roughness on the emittance of an electron beam, in Proceedings of the FEL 2006 Conference, Berlin, Germany (BESSY, Berlin, 2006), pp. 583-586.

[27] Y. Zou, H. Li, M. Reiser, and P. G. O'Shea, Theoretical study of transverse emittance growth in a gridded electron gun, Nucl. Instrum. Methods Phys. Res., Sect. A 519, 432 (2004). 